\title{
Noncommutative Classical and Quantum Mechanics for Quadratic Lagrangians (Hamiltonians)
}

\author{
Branko Dragovich* \\ Institute of Physics, P.O. Box 57, 11001 Belgrade, \\ Serbia and Montenegro \\ Zoran Rakić ${ }^{\dagger}$ \\ Faculty of Mathematics, P.O. Box 550, 11001 Belgrade, \\ Serbia and Montenegro
}

\begin{abstract}
We consider classical and quantum mechanics for an extended Heisenberg algebra with additional canonical commutation relations for position and momentum coordinates. In our approach this additional noncommutativity is removed from the algebra by linear transformation of coordinates and transmitted to the Hamiltonian (Lagrangian). Since linear transformations do not change the quadratic form of Hamiltonian (Lagrangian), and Feynman's path integral has well-known exact expression for quadratic models, we restricted our analysis to this class of physical systems. The compact general formalism presented here can be easily realized in any particular quadratic case. As an important example of phenomenological interest, we explored model of a charged particle in the noncommutative plane with perpendicular magnetic field. We also introduced an effective Planck constant $\hbar_{e f f}$ which depends on noncommutativity.
\end{abstract}

PACS numbers: 11.10.Nx, 03.65.Db, 03.65.-w

MSC2000: 81S40, 81T75, 81S10

Keywords: extended Heisenberg algebra, noncommutative quantum mechanics, path integrals, quadratic Lagrangians

* e-mail address: dragovich@phy.bg.ac.yu

$\dagger$ e-mail address: zrakic@matf.bg.ac.yu 


\section{Introduction}

It is well known that the standard $n$-dimensional quantum mechanics (QM) is based on the Heisenberg algebra

$$
\left[\hat{x}_{i}, \hat{p}_{j}\right]=i \hbar \delta_{i j}, \quad\left[\hat{x}_{i}, \hat{x}_{j}\right]=0, \quad\left[\hat{p}_{i}, \hat{p}_{j}\right]=0, \quad i, j=1,2, \cdots, n,
$$

which Hermitian operators for position and momentum coordinates satisfy in the Hilbert space of any standard quantum-mechanical system. However, in the last few years there has been an intensive interest in investigation of noncommutative QM (NCQM) which mainly starts from the commutation relations

$$
\left[\hat{x}_{i}, \hat{p}_{j}\right]=i \hbar \delta_{i j}, \quad\left[\hat{x}_{i}, \hat{x}_{j}\right]=i \hbar \theta_{i j}, \quad\left[\hat{p}_{i}, \hat{p}_{j}\right]=0, \quad i, j=1,2, \cdots, n,
$$

where $\theta_{i j}$ are constant elements of a real antisymmetric $\left(\theta_{i j}=-\theta_{j i}\right) n \times n$ matrix $\Theta$. Although the first considerations of noncommutativity (NC) (2) go back to the 1930's (see, e.g. [1]) the real excitement began in the 1998 when spatial $\mathrm{NC}$ of the form $\left[\hat{x}_{i}, \hat{x}_{j}\right]=i \hbar \theta_{i j}$ appeared in the low energy string theory with D-branes in a constant background B-field (see reviews [2], [3] and references therein).

Adopting the NC (2), one has wider than standard uncertainty, i.e.

$$
\Delta x_{i} \Delta p_{j} \geq \frac{\hbar}{2} \delta_{i j}, \quad \Delta x_{i} \Delta x_{j} \geq \frac{\hbar}{2}\left|\theta_{i j}\right|,
$$

which prevents from simultaneous accurate measuring not only usual $x_{i}$ and $p_{i}$ but also spatial coordinates $x_{i}$ and $x_{j} \quad(i \neq j)$. One often takes $\theta_{i j}=\theta \varepsilon_{i j}$, where $\left(\varepsilon_{i j}\right)=\mathcal{E}$ is the unit $n \times n$ antisymmetric matrix with $\varepsilon_{i j}=+1$ if $i<j$. Due to the uncertainty $\Delta x_{i} \Delta x_{j} \geq \frac{\hbar}{2}|\theta|, \quad(i \neq j)$, a spatial point is not a well defined concept and the space becomes fuzzy at distances of the order $\sqrt{\hbar|\theta|}$, which may be much larger than the Planck or string length.

Most portion of the research in this subject has been mainly devoted to noncommutative field theory (for reviews, see e.g. [2] and [3]). NCQM has been also actively explored. It is motivated by the fact that NCQM can be regarded as the corresponding one-particle nonrelativistic sector of noncommutative quantum field theory. Also it provides construction of noncommutative models suitable for the theoretical research and for potential experimental tests. To this end, many two-dimensional models have been studied and possible noncommutative contributions to the well-known effects (Aharonov-Bohm effect [4, lowest Landau level [1], fractional Hall effect [5], ...) have been investigated.

Evolution in NCQM has been mainly investigated using the Schrödinger equation. The path integral method has attracted less attention, however 
for systems with quadratic Lagrangians a systematic investigation started recently ( see [7]-9] and references therein).

We consider here $n$-dimensional NCQM which is mainly based on the following algebra

$$
\left[\hat{x_{a}}, \hat{p_{b}}\right]=i \hbar\left(\delta_{a b}-\frac{1}{4} \theta_{a c} \sigma_{c b}\right), \quad\left[\hat{x_{a}}, \hat{x_{b}}\right]=i \hbar \theta_{a b}, \quad\left[\hat{p_{a}}, \hat{p_{b}}\right]=i \hbar \sigma_{a b},
$$

where $\left(\theta_{a b}\right)=\Theta$ and $\left(\sigma_{a b}\right)=\Sigma$ are the antisymmetric matrices with constant elements. This kind of an extended noncommutativity maintains symmetry between canonical variables and yields (2) in the limit $\sigma_{a b} \rightarrow 0$. We suppose that $|\theta \sigma| \ll 1$. The algebra (4) allows simple reduction to the usual commutation relations

$$
\left[\hat{q_{a}}, \hat{k_{b}}\right]=i \hbar \delta_{a b}, \quad\left[\hat{q_{a}}, \hat{q_{b}}\right]=0, \quad\left[\hat{k_{a}}, \hat{k_{b}}\right]=0
$$

using the following linear transformations:

$$
\hat{x_{a}}=\hat{q_{a}}-\frac{\theta_{a b} \hat{k_{b}}}{2}, \quad \hat{p_{a}}=\hat{k_{a}}+\frac{\sigma_{a b} \hat{q_{b}}}{2},
$$

where summation over repeated indices is understood. It was shown recently 10] that NC (41) is suitable for study of possible dynamical control of decoherence by applying perpendicular magnetic field to a charged particle in the plane. This property also gives possibility to observe NC.

In this paper we present compact general formalism of NCQM for quadratic Lagrangians (Hamiltonians) with the above form of the NC. The formalism developed is suitable for both Schrödinger and Feynman approaches to quantum evolution. There are now many papers on some concrete models in NCQM, however, to our best knowledge, there is no article on the evaluation of general quadratic Lagrangians (Hamiltonians). Especially, Feynman's path integral method to the NC has been almost ignored. Note that quadratic Lagrangians contain an important class of physical models, and that some of them are rather simple and exactly solvable (a free particle, a particle in a constant field, a harmonic oscillator). The obtained relations between coefficients in commutative and noncommutative regimes give possibility to easily construct effective Hamiltonians and Lagrangians in the particular noncommutative cases.

Sec. 2 contains various expressions for quadratic Lagrangians and Hamiltonians in commutative and noncommutative regimes, as well as relations between them. In Sec. 3, the corresponding Schrödinger equation and Feynman path integral are written down. As an example of important phenomenological interest [10], a charged particle in the noncommutative plane with homogeneous perpendicular magnetic field is presented in Sec. 4. In the last section, we discuss and emphasize some main results. 


\section{Quadratic Lagrangians and Hamiltonians}

We start with general quadratic Lagrangian for an $n$-dimensional system, with position coordinates $x^{T}=\left(x_{1}, x_{2}, \cdots, x_{n}\right)$, which has the form

$$
L(\dot{x}, x, t)=\frac{1}{2}\left(\dot{x}^{T} \alpha \dot{x}+\dot{x}^{T} \beta x+x^{T} \beta^{T} \dot{x}+x^{T} \gamma x\right)+\delta^{T} \dot{x}+\eta^{T} x+\phi,
$$

where coefficients of the $n \times n$ matrices $\alpha=\left(\left(1+\delta_{a b}\right) \alpha_{a b}(t)\right)$, $\beta=\left(\beta_{a b}(t)\right), \gamma=\left(\left(1+\delta_{a b}\right) \gamma_{a b}(t)\right), n$-dimensional vectors $\delta=\left(\delta_{a}(t)\right)$, $\eta=\left(\eta_{a}(t)\right)$ and a scalar $\phi=\phi(t)$ are some analytic functions of the time $t$. Matrices $\alpha$ and $\gamma$ are symmetric, $\alpha$ is nonsingular ( $\operatorname{det} \alpha \neq 0$ ) and superscript $T$ denotes transposition.

The Lagrangian (17) can be rewritten in the more compact form:

$$
L(X, t)=\frac{1}{2} X^{T} M X+N^{T} X+\phi,
$$

where $2 n \times 2 n$ matrix $M$ and $2 n$-dimensional vectors $X, N$ are defined as

$$
M=\left(\begin{array}{cc}
\alpha & \beta \\
\beta^{T} & \gamma
\end{array}\right), \quad X^{T}=\left(\dot{x}^{T}, x^{T}\right), \quad N^{T}=\left(\delta^{T}, \eta^{T}\right) .
$$

Using the equations $p_{a}=\frac{\partial L}{\partial \dot{x}_{a}}$, one finds $\dot{x}=\alpha^{-1}(p-\beta x-\delta)$. Since the function $\dot{x}$ is linear in $p$ and $x$, the corresponding classical Hamiltonian $H(p, x, t)=p^{T} \dot{x}-L(\dot{x}, x, t)$ becomes also quadratic, i.e.

$H(p, x, t)=\frac{1}{2}\left(p^{T} A p+p^{T} B x+x^{T} B^{T} p+x^{T} C x\right)+D^{T} p+E^{T} x+F$

where:

$$
\begin{array}{lll}
A=\alpha^{-1}, & B=-\alpha^{-1} \beta, & C=\beta^{T} \alpha^{-1} \beta-\gamma, \\
D=-\alpha^{-1} \delta, & E=\beta^{T} \alpha^{-1} \delta-\eta, & F=\frac{1}{2} \delta^{T} \alpha^{-1} \delta-\phi .
\end{array}
$$

Due to the symmetry of matrices $\alpha$ and $\gamma$ one can easily see that matrices $A=\left(\left(1+\delta_{a b}\right) A_{a b}(t)\right)$ and $C=\left(\left(1+\delta_{a b}\right) C_{a b}(t)\right)$ are also symmetric $\left(A^{T}=\right.$ $\left.A, C^{T}=C\right)$. The nonsingular $(\operatorname{det} \alpha \neq 0$ ) Lagrangian $L(\dot{x}, x, t)$ implies nonsingular ( $\operatorname{det} A \neq 0$ ) Hamiltonian $H(p, x, t)$. Note that the inverse map, i.e. $H \rightarrow L$, is given by the same relations (11).

The Hamiltonian (10) can be also presented in the compact form 


$$
H(\Pi, t)=\frac{1}{2} \Pi^{T} \mathcal{M} \Pi+\mathcal{N}^{T} \Pi+F,
$$

where matrix $\mathcal{M}$ and vectors $\Pi, \mathcal{N}$ are

$$
\mathcal{M}=\left(\begin{array}{cc}
A & B \\
B^{T} & C
\end{array}\right), \quad \Pi^{T}=\left(p^{T}, x^{T}\right), \quad \mathcal{N}^{T}=\left(D^{T}, E^{T}\right) .
$$

One can show that

$$
\mathcal{M}=\sum_{i=1}^{3} \Upsilon_{i}^{T}(M) M \Upsilon_{i}(M)
$$

where

$$
\begin{array}{ll}
\Upsilon_{1}(M)=\left(\begin{array}{rr}
\alpha^{-1} & 0 \\
0 & -I
\end{array}\right), & \Upsilon_{2}(M)=\left(\begin{array}{rr}
0 & \alpha^{-1} \beta \\
0 & 0
\end{array}\right), \\
\Upsilon_{3}(M)=\left(\begin{array}{rr}
0 & 0 \\
0 & i \sqrt{2} I
\end{array}\right), &
\end{array}
$$

and $I$ is $n \times n$ unit matrix. One has also $\mathcal{N}=Y(M) N$, where

$$
Y(M)=\left(\begin{array}{rr}
-\alpha^{-1} & 0 \\
\beta^{T} \alpha^{-1} & -I
\end{array}\right)=-\Upsilon_{1}(M)+\Upsilon_{2}^{T}(M)+i \sqrt{2} \Upsilon_{3}(M),
$$

and $F=N^{T} Z(M) N-\phi$, where

$$
Z(M)=\left(\begin{array}{ll}
\frac{1}{2} \alpha^{-1} & 0 \\
0 & 0
\end{array}\right)=\frac{1}{2} \Upsilon_{1}(M)-\frac{i}{2 \sqrt{2}} \Upsilon_{3}(M) .
$$

Using auxiliary matrices $\Upsilon_{1}(M), \Upsilon_{2}(M)$ and $\Upsilon_{3}(M)$ in the above way, Hamiltonian quantities $\mathcal{M}, \mathcal{N}$ and $F$ are connected to the corresponding Lagrangian ones $M, N$ and $\phi$.

Eqs. (66) can be rewritten in the compact form as

$$
\hat{\Pi}=\Xi \hat{K}, \quad \Xi=\left(\begin{array}{cc}
I & \frac{1}{2} \Sigma \\
-\frac{1}{2} \Theta & I
\end{array}\right), \quad \hat{K}=\left(\begin{array}{c}
\hat{k} \\
\hat{q}
\end{array}\right) .
$$


Since Hamiltonians depend on canonical variables, the transformation (18) leads to the transformation of Hamiltonians (10) and (12). To this end, let us quantize the Hamiltonian (10) and it easily becomes

$$
H(\hat{p}, \hat{x}, t)=\frac{1}{2}\left(\hat{p}^{T} A \hat{p}+\hat{p}^{T} B \hat{x}+\hat{x}^{T} B^{T} \hat{p}+\hat{x}^{T} C \hat{x}\right)+D^{T} \hat{p}+E^{T} \hat{x}+F
$$

because (10) is already written in the Weyl symmetric form.

Performing linear transformations (6) in (19) we again obtain quadratic quantum Hamiltonian

$$
\begin{aligned}
H_{\theta \sigma}(\hat{k}, \hat{q}, t)= & \frac{1}{2}\left(\hat{k}^{T} A_{\theta \sigma} \hat{k}+\hat{k}^{T} B_{\theta \sigma} \hat{q}+\hat{q}^{T} B_{\theta \sigma}^{T} \hat{k}+\hat{q}^{T} C_{\theta \sigma} \hat{q}\right) \\
& +D_{\theta \sigma}^{T} \hat{k}+E_{\theta \sigma}^{T} \hat{q}+F_{\theta \sigma},
\end{aligned}
$$

where

$$
\begin{array}{ll}
A_{\theta \sigma}=A-\frac{1}{2} B \Theta+\frac{1}{2} \Theta B^{T}-\frac{1}{4} \Theta C \Theta, & D_{\theta \sigma}=D+\frac{1}{2} \Theta E \\
B_{\theta \sigma}=B+\frac{1}{2} \Theta C+\frac{1}{2} A \Sigma+\frac{1}{4} \Theta B^{T} \Sigma, & E_{\theta \sigma}=E-\frac{1}{2} \Sigma D, \\
C_{\theta \sigma}=C-\frac{1}{2} \Sigma B+\frac{1}{2} B^{T} \Sigma-\frac{1}{4} \Sigma A \Sigma, & F_{\theta \sigma}=F .
\end{array}
$$

Note that for the nonsingular Hamiltonian $H(\hat{p}, \hat{x}, t)$ and for sufficiently small $\theta_{a b}$ the Hamiltonian $H_{\theta \sigma}(\hat{k}, \hat{q}, t)$ is also nonsingular. $A_{\theta \sigma}$ and $D_{\theta \sigma}$ do not depend on $\sigma$, as well as $C_{\theta \sigma}$ and $E_{\theta \sigma}$ do not contain $\theta$.

It is worth noting that Hamiltonian (20) with noncommutativity (41) is equivalent to Hamiltonian (21) with ordinary commutation relations (15). Dynamics depends on parameters $\theta$ and $\sigma$, what is more obvious from Hamiltonian (20) than relations (41). Consequently phase space transformations (6) are not (and should not be) canonical, because dynamics of our system is given not only by Hamiltonian (20) but with (20) and (4). In the case $\sigma=0$, a similar result for Hamiltonian with parameter $\theta$ is obtained by the Moyal product procedure 6].

Classical analogue of (20) maintains the same form

$$
\begin{aligned}
H_{\theta \sigma}(k, q, t)= & \frac{1}{2}\left(k^{T} A_{\theta \sigma} k+k^{T} B_{\theta \sigma} q+q^{T} B_{\theta \sigma}^{T} k+q^{T} C_{\theta \sigma} q\right) \\
& +D_{\theta \sigma}^{T} k+E_{\theta \sigma}^{T} q+F_{\theta \sigma} .
\end{aligned}
$$


In the compact form Hamiltonian (20) becomes

$$
\hat{H}_{\theta \sigma}(\hat{K}, t)=\frac{1}{2} \hat{K}^{T} \mathcal{M}_{\theta \sigma} \hat{K}+\mathcal{N}_{\theta \sigma}^{T} \hat{K}+F_{\theta \sigma}
$$

where $2 n \times 2 n$ matrix $\mathcal{M}_{\theta \sigma}$ and $2 n$-dimensional vectors $\mathcal{N}_{\theta \sigma}, \hat{K}$ are

$$
\mathcal{M}_{\theta \sigma}=\left(\begin{array}{cc}
A_{\theta \sigma} & B_{\theta \sigma} \\
B_{\theta \sigma}^{T} & C_{\theta \sigma}
\end{array}\right), \quad \mathcal{N}_{\theta \sigma}^{T}=\left(D_{\theta \sigma}^{T}, E_{\theta \sigma}^{T}\right), \quad \hat{K}^{T}=\left(\hat{k}^{T}, \hat{q}^{T}\right)
$$

From (12), (18) and (22) one can find connections between $\mathcal{M}_{\theta \sigma}, \mathcal{N}_{\theta \sigma}, F_{\theta \sigma}$ and $\mathcal{M}, \mathcal{N}, F$, which are given by the following relations:

$$
\mathcal{M}_{\theta \sigma}=\Xi^{T} \mathcal{M} \Xi, \quad \mathcal{N}_{\theta \sigma}=\Xi^{T} \mathcal{N}, \quad F_{\theta \sigma}=F .
$$

Using equations $\dot{q}_{a}=\frac{\partial H_{\theta \sigma}}{\partial k_{a}}$ which give $k=A_{\theta \sigma}^{-1}\left(\dot{q}-B_{\theta \sigma} q-D_{\theta \sigma}\right)$, we can pass from the classical form of Hamiltonian (20) to the corresponding Lagrangian by relation $L_{\theta \sigma}(\dot{q}, q, t)=k^{T} \dot{q}-H_{\theta \sigma}(k, q, t)$. Note that coordinates $q_{a}$ and $x_{a}$ coincide when $\theta=\sigma=0$. Performing necessary computations we obtain

$$
\begin{aligned}
L_{\theta \sigma}(\dot{q}, q, t)= & \frac{1}{2}\left(\dot{q}^{T} \alpha_{\theta \sigma} \dot{q}+\dot{q}^{T} \beta_{\theta \sigma} q+q^{T} \beta_{\theta \sigma}^{T} \dot{q}+q^{T} \gamma_{\theta \sigma} q\right) \\
& +\delta_{\theta \sigma}^{T} \dot{q}+\eta_{\theta \sigma}^{T} q+\phi_{\theta \sigma},
\end{aligned}
$$

or in the compact form:

$$
L_{\theta \sigma}(Q, t)=\frac{1}{2} Q^{T} M_{\theta \sigma} Q+N_{\theta \sigma}^{T} Q+\phi_{\theta \sigma},
$$

where

$$
M_{\theta \sigma}=\left(\begin{array}{cc}
\alpha_{\theta \sigma} & \beta_{\theta \sigma} \\
\beta_{\theta \sigma}^{T} & \gamma_{\theta \sigma}
\end{array}\right), \quad N_{\theta \sigma}^{T}=\left(\delta_{\theta \sigma}^{T}, \eta_{\theta \sigma}^{T}\right), \quad Q^{T}=\left(\dot{q}^{T}, q^{T}\right), .
$$

Then the connections between $M_{\theta \sigma}, N_{\theta \sigma}, \phi_{\theta \sigma}$ and $M, N, \phi$ are given by the following relations:

$$
\begin{array}{ll}
M_{\theta \sigma}=\sum_{i, j=1}^{3} \Xi_{i j}^{T} M \Xi_{i j}, & \Xi_{i j}=\Upsilon_{i}(M) \Xi \Upsilon_{j}\left(\mathcal{M}_{\theta \sigma}\right), \\
N_{\theta \sigma}=Y\left(\mathcal{M}_{\theta \sigma}\right) \Xi^{T} Y(M) N, & \phi_{\theta \sigma}=\mathcal{N}_{\theta \sigma}^{T} Z\left(\mathcal{M}_{\theta \sigma}\right) \mathcal{N}_{\theta \sigma}-F .
\end{array}
$$


In more detail, the connection between coefficients of the Lagrangians $L_{\theta \sigma}$ and $L$ is given by the relations:

$$
\begin{aligned}
\alpha_{\theta \sigma}= & {\left[\alpha^{-1}-\frac{1}{2}\left(\Theta \beta^{T} \alpha^{-1}-\alpha^{-1} \beta \Theta\right)-\frac{1}{4} \Theta\left(\beta^{T} \alpha^{-1} \beta-\gamma\right) \Theta\right]^{-1}, } \\
\beta_{\theta \sigma}= & \alpha_{\theta \sigma}\left(\alpha^{-1} \beta-\frac{1}{2}\left(\alpha^{-1} \Sigma-\Theta \gamma+\Theta \beta^{T} \alpha^{-1} \beta\right)+\frac{1}{4} \Theta \beta^{T} \alpha^{-1} \Sigma\right), \\
\gamma_{\theta \sigma}= & \gamma+\beta_{\theta \sigma}^{T} \alpha_{\theta \sigma}^{-1} \beta_{\theta \sigma}-\beta^{T} \alpha^{-1} \beta+\frac{1}{4} \Sigma \alpha^{-1} \Sigma \\
& -\frac{1}{2}\left(\Sigma \alpha^{-1} \beta-\beta^{T} \alpha^{-1} \Sigma\right), \\
\delta_{\theta \sigma}= & \alpha_{\theta \sigma}\left(\alpha^{-1} \delta+\frac{1}{2}\left(\Theta \eta-\Theta \beta^{T} \alpha^{-1} \delta\right)\right), \\
\eta_{\theta \sigma}= & \eta+\beta_{\theta \sigma}^{T} \alpha_{\theta \sigma}^{-1} \delta_{\theta \sigma}-\beta^{T} \alpha^{-1} \delta-\frac{1}{2} \Sigma \alpha^{-1} \delta, \\
\phi_{\theta \sigma}= & \phi+\frac{1}{2} \delta_{\theta \sigma}^{T} \alpha_{\theta \sigma}^{-1} \delta_{\theta \sigma}-\frac{1}{2} \delta^{T} \alpha^{-1} \delta .
\end{aligned}
$$

Note that $\alpha_{\theta \sigma}, \delta_{\theta \sigma}$ and $\phi_{\theta \sigma}$ do not depend on $\sigma$.

\section{Noncommutative Schrödinger Equation and Path Integral}

The corresponding Schrödinger equation in this NCQM is

$$
i \hbar \frac{\partial \Psi(q, t)}{\partial t}=H_{\theta \sigma}(\hat{k}, q, t) \Psi(q, t)
$$

where $\hat{k}_{a}=-i \hbar \frac{\partial}{\partial q_{a}}, a=1,2, \cdots, n$ and $H_{\theta \sigma}(\hat{k}, q, t)$ is given by (20). Investigations of dynamical evolution have been mainly performed using the Schrödinger equation and this aspect of NCQM is much more developed than the noncommutative Feynman path integral. For this reason and importance of the path integral method, we will in the sequel give priority to the description of this approach.

To compute a path integral, which is a basic instrument in Feynman's approach to quantum mechanics, one can start from its Hamiltonian formulation on the phase space. However, when Hamiltonian is a quadratic polynomial with respect to momentum $k$ (see, e.g. 8] ) such path integral on a phase space can be reduced to the Lagrangian path integral on configuration space. Hence, for the Hamiltonians (20) and (22) we have derived the corresponding Lagrangians (25) and (26). 
The standard Feynman path integral [11] is

$$
\mathcal{K}\left(x^{\prime \prime}, t^{\prime \prime} ; x^{\prime}, t^{\prime}\right)=\int_{x^{\prime}}^{x^{\prime \prime}} \exp \left(\frac{i}{\hbar} \int_{t^{\prime}}^{t^{\prime \prime}} L(\dot{q}, q, t) d t\right) \mathcal{D} q
$$

where $\mathcal{K}\left(x^{\prime \prime}, t^{\prime \prime} ; x^{\prime}, t^{\prime}\right)$ is the kernel of the unitary evolution operator $U(t)$ and $x^{\prime \prime}=q\left(t^{\prime \prime}\right), x^{\prime}=q\left(t^{\prime}\right)$ are end points. In ordinary quantum mechanics (OQM), Feynman's path integral for quadratic Lagrangians can be evaluated analytically and its exact expression has the form [12]

$$
\mathcal{K}\left(x^{\prime \prime}, t^{\prime \prime} ; x^{\prime}, t^{\prime}\right)=\frac{1}{(i h)^{\frac{n}{2}}} \sqrt{\operatorname{det}\left(-\frac{\partial^{2} \bar{S}}{\partial x_{a}^{\prime \prime} \partial x_{b}^{\prime}}\right)} \exp \left(\frac{2 \pi i}{h} \bar{S}\left(x^{\prime \prime}, t^{\prime \prime} ; x^{\prime}, t^{\prime}\right)\right),
$$

where $\bar{S}\left(x^{\prime \prime}, t^{\prime \prime} ; x^{\prime}, t^{\prime}\right)$ is the action for the classical trajectory. According to (44), (5) and (6), NCQM related to the quantum phase space $(\hat{p}, \hat{x})$ can be regarded as an OQM on the standard phase space $(\hat{k}, \hat{q})$ and one can apply usual path integral formalism.

A systematic path integral approach to NCQM with quadratic Lagrangians (Hamiltonians) has been investigated during the last few years in [7], 8] and 9]. In [7] and [8, general connections between quadratic Lagrangians and Hamiltonians for standard and $\theta \neq 0, \sigma=0 \mathrm{NC}$ are established, and this formalism was applied to a particle in the two-dimensional noncommutative plane with a constant field and to the noncommutative harmonic oscillator. Paper 9] presents generalization of articles [7] and [8] towards noncommutativity (4). The present article develops formalism of [9] and contains its application to a charged particle in the noncommutative plane with homogeneous electric and perpendicular magnetic field.

If we know Lagrangian (77) and algebra (44) we can obtain the corresponding effective Lagrangian (25) suitable for the path integral in NCQM. Exploiting the Euler-Lagrange equations

$$
\frac{\partial L_{\theta \sigma}}{\partial q_{a}}-\frac{d}{d t} \frac{\partial L_{\theta \sigma}}{\partial \dot{q}_{a}}=0, \quad a=1,2, \cdots, n,
$$

one can obtain the classical trajectory $q_{a}=q_{a}(t)$ connecting end points $x^{\prime}=q\left(t^{\prime}\right)$ and $x^{\prime \prime}=q\left(t^{\prime \prime}\right)$, and the corresponding action is

$$
\bar{S}_{\theta \sigma}\left(x^{\prime \prime}, t^{\prime \prime} ; x^{\prime}, t^{\prime}\right)=\int_{t^{\prime}}^{t^{\prime \prime}} L_{\theta \sigma}(\dot{q}, q, t) d t
$$

Path integral in NCQM is a direct analogue of (32) and its exact expression in the form of quadratic actions $\bar{S}_{\theta \sigma}\left(x^{\prime \prime}, t^{\prime \prime} ; x^{\prime}, t^{\prime}\right)$ is

$$
\mathcal{K}_{\theta \sigma}\left(x^{\prime \prime}, t^{\prime \prime} ; x^{\prime}, t^{\prime}\right)=\frac{1}{(i h)^{\frac{n}{2}}} \sqrt{\operatorname{det}\left(-\frac{\partial^{2} \bar{S}_{\theta \sigma}}{\partial x_{a}^{\prime \prime} \partial x_{b}^{\prime}}\right)} \exp \left(\frac{2 \pi i}{h} \bar{S}_{\theta \sigma}\left(x^{\prime \prime}, t^{\prime \prime} ; x^{\prime}, t^{\prime}\right)\right) \text {. }
$$




\section{Particle in a Noncommutative Plane with Electric and Perpendicular Magnetic Field}

To illustrate many features of the above formalism, as well as to give a model suitable for potential phenomenology (see, e.g. [10]), we consider a particle of a charge $e$ moving in a plane $\left(x_{1}, x_{2}\right)$ with noncommutativity parameters $\theta$ and $\sigma$. Let this particle be also under the influence of a homogeneous electric field $\mathcal{E}$ along coordinate $x_{1}$ and a homogeneous magnetic field $\mathcal{B}$ perpendicular to the plane and oriented along the axis $-x_{3}$. It is convenient to start from the nonrelativistic Hamiltonian

$$
H(p, x)=\frac{1}{2 m}\left[\left(p_{1}-e \mathcal{A}_{1}\right)^{2}+\left(p_{2}-e \mathcal{A}_{2}\right)^{2}\right]+e \varphi,
$$

where $\mathcal{A}_{1}=\frac{\mathcal{B}}{2} x_{2}, \mathcal{A}_{2}=-\frac{\mathcal{B}}{2} x_{1}$ and $\varphi=-\mathcal{E} x_{1}$. This dynamical system is also very suitable to study possible phenomenological aspects of NCQM. Using the inverse map of (11) we get the corresponding Lagrangian

$$
L(\dot{x}, x)=\frac{m}{2}\left(\dot{x}_{1}^{2}+\dot{x}_{2}^{2}\right)+\frac{e \mathcal{B}}{2}\left(\dot{x}_{1} x_{2}-\dot{x}_{2} x_{1}\right)+e \mathcal{E} x_{1} .
$$

Employing formulas (21) and (29), one obtains respectively Hamiltonian

$$
\begin{aligned}
H_{\theta \sigma}(k, q)= & \frac{1}{2 \mu}\left(k_{1}^{2}+k_{2}^{2}\right)-\frac{\lambda}{2 \mu}\left(k_{1} q_{2}-k_{2} q_{1}\right)+\frac{\lambda^{2}}{8 \mu}\left(q_{1}^{2}+q_{2}^{2}\right) \\
& +\frac{\theta e \mathcal{E}}{2} k_{2}-e \mathcal{E} q_{1}
\end{aligned}
$$

and Lagrangian

$$
L_{\theta \sigma}(\dot{q}, q)=\frac{\mu}{2}\left(\dot{q}_{1}^{2}+\dot{q}_{2}^{2}\right)+\frac{\lambda}{2}\left(\dot{q}_{1} q_{2}-\dot{q}_{2} q_{1}\right)-\frac{\mu \theta e \mathcal{E}}{2} \dot{q}_{2}+\nu_{0} q_{1}+\frac{\mu \theta^{2} e^{2} \mathcal{E}^{2}}{8},(37
$$

where

$$
\mu=\frac{m}{\left(1-\frac{\theta e \mathcal{B}}{4}\right)^{2}}, \quad \lambda=\frac{e \mathcal{B}-\sigma}{1-\frac{\theta e \mathcal{B}}{4}}, \quad \nu_{0}=e \mathcal{E}\left(1+\frac{\theta \lambda}{4}\right) .
$$


The above Hamiltonian and Lagrangian are related to the dynamics in noncommutative plane, where $\mathrm{NC}$ is characterized by parameters $\theta$ and $\sigma$.

The Lagrangian given by (37) implies the Euler-Lagrange equations,

$$
\mu \ddot{q}_{1}+\lambda \dot{q}_{2}=\nu_{0}, \quad \mu \ddot{q}_{2}-\lambda \dot{q}_{1}=0 .
$$

We transform the system (39) to two uncoupled third order differential equations

$$
\mu^{2} q_{1}^{(3)}+\lambda^{2} q_{1}^{(1)}=0, \quad \quad \mu^{2} q_{2}^{(3)}+\lambda^{2} q_{2}^{(1)}-\lambda \nu_{0}=0 .
$$

The solution of the equations (40) has the following form

$$
\begin{aligned}
& q_{1}(t)=C_{1}+C_{2} \cos (\eta t)+C_{3} \sin (\eta t), \\
& q_{2}(t)=D_{1}+D_{2} \cos (\eta t)+D_{3} \sin (\eta t)+\frac{\nu_{0}}{\lambda} t, \quad \eta=\frac{\lambda}{\mu} .
\end{aligned}
$$

Imposing connection between $q_{1}$ and $q_{2}$ by coupled differential equations (39), we obtain $C_{2}=D_{3}$ and $D_{2}=-C_{3}$. The constants $C_{1}, D_{1}, C_{3}$ and $D_{3}$ can be fixed from initial conditions given by

$$
q_{1}(0)=x_{1}^{\prime}, \quad q_{1}(T)=x_{1}^{\prime \prime}, \quad q_{2}(0)=x_{2}^{\prime}, \quad q_{2}(T)=x_{2}^{\prime \prime} .
$$

By this way the corresponding constants become :

$$
\begin{aligned}
C_{1} & =\frac{x_{1}^{\prime}+x_{1}^{\prime \prime}}{2}+\frac{x_{2}^{\prime}-x_{2}^{\prime \prime}}{2} \cot \left[\frac{T \eta}{2}\right]+\frac{T \nu_{0}}{2 \lambda} \cot \left[\frac{T \eta}{2}\right], \\
C_{3} & =\frac{-x_{2}^{\prime}+x_{2}^{\prime \prime}}{2}-\frac{x_{1}^{\prime}-x_{1}^{\prime \prime}}{2} \cot \left[\frac{T \eta}{2}\right]-\frac{T \nu_{0}}{2 \lambda}, \\
D_{1} & =\frac{x_{2}^{\prime}+x_{2}^{\prime \prime}}{2}-\frac{x_{1}^{\prime}-x_{1}^{\prime \prime}}{2} \cot \left[\frac{T \eta}{2}\right]-\frac{T \nu_{0}}{2 \lambda}, \\
D_{3} & =\frac{x_{1}^{\prime}-x_{1}^{\prime \prime}}{2}-\frac{x_{2}^{\prime}-x_{2}^{\prime \prime}}{2} \cot \left[\frac{T \eta}{2}\right]-\frac{T \nu_{0}}{2 \lambda} \cot \left[\frac{T \eta}{2}\right] .
\end{aligned}
$$

Inserting the above expressions for constants (43) into (41) we obtain solutions of the Euler-Lagrange equations (39). For these solutions and their time derivatives we find the following expression for Lagrangian (37):

$$
\begin{aligned}
& L_{\theta \sigma}(\dot{q}, q)=\frac{1}{8 \lambda^{2} \mu}\left(\mu\left(4 C_{1} \lambda^{2} \nu_{0}+\mu\left(-2 \nu_{0}+\theta \lambda e \mathcal{E}\right)^{2}\right)+4 \lambda^{2}\right. \\
& \quad \times\left(\left(C_{3} \lambda\left(D_{1} \lambda+\nu_{0} t\right)-D_{3}\left(C_{1} \lambda^{2}-3 \mu \nu_{0}+\theta \lambda \mu e \mathcal{E}\right)\right) \cos [\eta t]\right. \\
& \left.\left.\quad-\left(\left(C_{1} C_{3}+D_{1} D_{3}\right) \lambda^{2}+D_{3} \lambda \nu_{0} t-3 C_{3} \mu \nu_{0}+C_{3} \theta \lambda \mu e \mathcal{E}\right) \sin [\eta t]\right)\right) .
\end{aligned}
$$


Using (44), we finally compute the corresponding classical action

$$
\begin{gathered}
\bar{S}_{\theta \sigma}\left(x^{\prime \prime}, T ; x^{\prime}, 0\right)=\int_{0}^{T} L_{\theta \sigma}(\dot{q}, q) d t=\frac{\lambda}{2}\left(x_{2}^{\prime} x_{1}^{\prime \prime}-x_{1}^{\prime} x_{2}^{\prime \prime}\right)+\frac{\nu_{0} T}{2}\left(x_{1}^{\prime}+x_{1}^{\prime \prime}\right) \\
+\frac{\mu \nu_{0}}{\lambda}\left(x_{2}^{\prime \prime}-x_{2}^{\prime}\right)+\frac{\theta \mu e \mathcal{E}}{2}\left(x_{2}^{\prime}-x_{2}^{\prime \prime}\right)+\frac{\mu T}{8}\left(-4 \frac{\nu_{0}^{2}}{\lambda^{2}}+\theta^{2} e^{2} \mathcal{E}^{2}\right) \\
+\frac{\left(x_{1}^{\prime}-x_{1}^{\prime \prime}\right)^{2} \lambda^{2}+\left(\left(x_{2}^{\prime}-x_{2}^{\prime \prime}\right) \lambda+T \nu_{0}\right)^{2}}{4 \lambda} \cot \left[\frac{\eta t}{2}\right] .
\end{gathered}
$$

Finally, we obtain the determinant

$$
\operatorname{det}\left(-\frac{\partial^{2} \bar{S}_{\theta \sigma}}{\partial x_{a}^{\prime \prime} \partial x_{b}^{\prime}}\right)=\frac{\lambda^{2}}{4 \sin ^{2}\left[\frac{\lambda T}{2}\right]}
$$

and the transition probability amplitude to reach the point $\left(x^{\prime \prime}, T\right)$ from the point $\left(x^{\prime}, 0\right)$ :

$$
\mathcal{K}_{\theta \sigma}\left(x^{\prime \prime}, T ; x^{\prime}, 0\right)=\frac{|\lambda|}{2 i h\left|\sin \left[\frac{\lambda T}{2}\right]\right|} \exp \left(\frac{2 \pi i}{h} \bar{S}_{\theta \sigma}\left(x^{\prime \prime}, T ; x^{\prime}, 0\right)\right),
$$

where $\bar{S}_{\theta \sigma}\left(x^{\prime \prime}, T ; x^{\prime}, 0\right)$ is given by (45). According to the Feynman approach to QM, all information on our noncommutative quantum system is encoded in the $\mathcal{K}_{\theta \sigma}\left(x^{\prime \prime}, T ; x^{\prime}, 0\right)$ in (47).

\section{Discussion and Concluding Remarks}

Let us mention that taking $\sigma=0, \theta=0$ in the above formulas we recover expressions for the Lagrangian $L(\dot{q}, q)$, classical action $\bar{S}\left(x^{\prime \prime}, T ; x^{\prime}, 0\right)$ and probability amplitude $\mathcal{K}\left(x^{\prime \prime}, T ; x^{\prime}, 0\right)$ of the ordinary commutative case.

Note that a similar path integral approach with $\sigma=0$ has been considered in the context of the Aharonov-Bohm effect 4, the Casimir effect, a quantum system in a rotating frame [13, and the Hall effect [5] ( see also reherences in [7, 8, 9]). Our investigation contains all quantum-mechanical systems with quadratic Hamiltonians (19) (Lagrangians (7)) on noncommutative phase space given by (4).

On the basis of the expressions presented in this article, there are many possibilities to discuss noncommutative quantum-mechanical systems with respect to various values of noncommutativity parameters $\theta$ and $\sigma$. We will 
discuss only some aspects of the two-dimensional model from the preceding section taking electric field $\mathcal{E}=0$.

Since

$$
\left[\hat{\pi}_{a}, \hat{\pi}_{b}\right]=-i \hbar(e \mathcal{B}-\sigma)\left(1-\frac{e \mathcal{B} \theta}{4}\right) \varepsilon_{a b}
$$

where $\hat{\pi}_{a}=\hat{p}_{a}-e \hat{\mathcal{A}}_{a}$, a charged particle in the plane with the homogeneous perpendicular magnetic field $\mathcal{B}$ and phase space NC (4) has motion under an effective momentum $\mathrm{NC}$ depending on $-(e \mathcal{B}-\sigma)\left(1-\frac{e \mathcal{B} \theta}{4}\right)$. Recall that $p$ is a canonical momentum and $\pi$ is the corresponding physical one.

Note that the NC parameter $\sigma$ appears in the form $e \mathcal{B}-\sigma$, i.e. $\sigma$ and $e \mathcal{B}$ are on the equal footing. Accordingly, magnetic field $\mathcal{B}$ can be regarded as a generator of the momentum NC with $\sigma=-e \mathcal{B}$. In our galaxy and other galaxies, there is evidence (see, e.g. [14] and [15]) of magnetic field $\mathcal{B} \sim$ $10^{-10} \mathrm{~T}, \quad(T=$ Tesla $)$ which may generate an effective $\sigma \sim 10^{-29} C T,(C=$ Coulomb), relevant to dynamics of electrons and protons. In the expression $e \mathcal{B}-\sigma$ one can regard $\sigma$ as a result of an effective background magnetic field and $e \mathcal{B}$ as the effect of a laboratory magnetic field.

It is also worth noting that there is a sense to introduce an effective reduced Planck constant as

$$
\hbar_{e f f}=\hbar\left(1+\frac{\theta \sigma}{4}\right)
$$

where one can take $\sigma=-e \mathcal{B}$. The present values of $\theta$ and $\sigma$ are very small so that $\frac{\theta \sigma}{4} \ll 1$ and consequently $\hbar_{e f f} \approx \hbar$, but at very early times of the universe evolution the situation might be very different. Suppose that at least one of parameters $\theta$ and $\sigma$ depends on cosmic time and let at very early stage of the evolution $\frac{\theta \sigma}{4} \gg 1$ and after that it decreased to a small value rapidly. Then $\frac{\theta \sigma}{4} \gg 1$ imposes $\hbar_{e f f} \gg \hbar$ and the universe was in a strengthened quantum state, which could significantly change our view of its beginning. It follows that existence of $\theta \neq 0$ and $\sigma \neq 0$, where the corresponding $\sigma$ can be generated by magnetic field, may modify $\hbar$, and this should play also some role at very high energies.

Using annihilation and creation operators

$$
a=\frac{\hat{\pi}_{1}-i \hat{\pi_{2}}}{\sqrt{2 \hbar_{e f f} m \omega}}, \quad a^{\dagger}=\frac{\hat{\pi}_{1}+i \hat{\pi}_{2}}{\sqrt{2 \hbar_{e f f} m \omega}},
$$

where the frequency

$$
\omega=\frac{e \mathcal{B}-\sigma}{m}
$$


one can write the Hamiltonian (136), with noncommutative phase space (4) and the electric field $\mathcal{E}=0$, in the harmonic oscillator form

$$
\hat{H}=\omega \hbar_{e f f}\left(a^{\dagger} a+\frac{1}{2}\right)
$$

with energy levels $E_{n}=\omega \hbar_{e f f}\left(n+\frac{1}{2}\right)$, where now $\hbar_{e f f}=\hbar\left(1-\frac{e \mathcal{B} \theta}{4}\right)$. This is an extended Landau problem which reduces to the standard one if $\sigma=\theta=0$. The frequency $\omega$ can be at will controlled by magnetic field $\mathcal{B}$. In particular, $\omega=0$ if $\mathcal{B}=\frac{\sigma}{e}$, and $\omega_{\mathcal{B} \rightarrow 0}=-\frac{\sigma}{m}$ as well as $\omega=\frac{e \mathcal{B}}{m}$ if $e \mathcal{B} \gg \sigma$.

In the very strong magnetic field $\mathcal{B}$ and $\mathcal{E}=0$, the Lagrangian (37) becomes

$$
L=\frac{2}{\theta}\left(\dot{x}_{2} x_{1}-\dot{x}_{1} x_{2}\right)
$$

The corresponding canonical momentum is $p_{1}=\frac{\partial L}{\partial \dot{x}_{1}}=-\frac{2}{\theta} x_{2}$ and

$$
\left[\hat{x}_{1}, \hat{p}_{1}\right]=\left[\hat{x}_{1},-\frac{2}{\theta} \hat{x}_{2}\right]=i \hbar_{e f f}
$$

In this limit one has a modified noncommutative configuration space with $\left[\hat{x}_{1}, \hat{x}_{2}\right]=-i \hbar_{\text {eff }} \frac{\theta}{2}$. The spacing between energy levels diverges like $-\frac{\hbar e^{2} \mathcal{B}^{2} \theta}{4 m}$ and the system practically lives in the lowest level $-\frac{\hbar e^{2} \mathcal{B}^{2} \theta}{8 m}$, which differs from the standard Landau lowest level $\frac{\hbar e \mathcal{B}}{2 m}$.

Summarizing, the main results of this paper are:

(i) compact presentation of classical and quantum mechanics related to the phase space $\mathrm{NC}(4)$;

(ii) explicit formulae (29) for coefficients of quadratic Lagrangian in noncommutative regime with respect to the coefficients of commutative one;

(iii) general expressions of the Schrödinger equation (30) and the Feynman path integral (33) for noncommutative quadratic Hamiltonian (20) and Lagrangian (25);

(iv) Feynman path integral (47) and energy spectrum (52) for a charged particle in the noncommutative plane with perpendicular magnetic field; and

(v) introduction of the effective noncommutative Planck constant (49), which could have significant cosmological implications.

At the end, it is worth mentioning that there are some other commutation relations similar to (4), which by linear transformations of canonical variables can be converted to the usual Heisenberg algebra (5). This will be presented elsewhere. 


\section{Acknowledgments}

The work on this article was partially supported by the Ministry of Science and Environmental Protection, Serbia, under contract No 144032D.

\section{References}

[1] R.J. Szabo, Int. J. Mod. Phys. A 19 (2004) 1837 - 1862.

[2] M. R. Douglas and N. A. Nekrasov, Rev. Mod. Phys. 73 (2001) 977 1029 .

[3] R.J. Szabo, Phys. Rep. 378 (2003) 207 - 299.

[4] M. Chaichian, A. Demichev, P. Prešnajder, M.M. Sheikh-Jabbari and A. Tureanu, Phys. Lett. B 527 (2002) 149 -154.

[5] Ö.F. Dayi and A. Jellal, J. Math. Phys. 43 (2002) 4592 - 4601.

[6] L. Mezincescu, Star operation in Quantum Mechanics, hep-th/0007046.

[7] B. Dragovich and Z. Rakić, Lagrangian Aspects of Quantum Dynamics on a Noncommutative Space, in Proc. of the Workshop "Contemporery Geometry and Related Topics" Belgrade, May 2004, edited by N. Bokan et al. (World Scientific, Singapore, 2004), 159 - 171, hep-th/0302167.

[8] B. Dragovich and Z. Rakić, Theor. Math. Phys. 140 (2004) 1299 - 1308, hep-th/0309204.

[9] B. Dragovich and Z. Rakić, Path Integral Approach to Noncommutative Quantum Mechanics, in Proc. of the Fifth International Workshop " Lie Theory and its Applications in Physics", Varna, Bulgaria, June 2003, edited by H.-D. Doebner and V.K. Dobrev (World Scientific, Singapore, 2004), 364 - 373 , hep-th/0401198 ; Noncommutative Quantum Mechanics with Path Integral, hep-th/0501231.

[10] B. Dragovich and M. Dugić, J. Phys. A: Math. Gen. 38 (2005) 66036611, quant-ph/0503163

[11] R. P. Feynman and A. R. Hibbs, Quantum Mechanics and Path Integrals, (McGraw - Hill Book Company, New York, 1965).

[12] C. Grosche and F. Steiner, Handbook of Feynman Path Integrals (Springer - Verlag, Berlin, 1998). 
[13] H.R. Christiansen and F.A. Schaposnik, Phys. Rev. D 65 (2002) 086005.

[14] K. Bamba and J. Yokoyama, Generation of Large-Scale Magnetic Fields from Dilaton Inflation in Noncommutative Spacetime, hep-ph/0502244.

[15] L.M. Widrow, Rev. Mod. Phys. 74 (2002) 775 - 823. 